\title{
Robust Sliding Mode Control for Nonlinear Discrete-Time Delayed Systems Based on Neural Network
}

\author{
Vishal Goyal, Vinay Kumar Deolia, Tripti Nath Sharma \\ Department of Electronics and Communication Engineering, G. L. A. University, Mathura, India \\ Email: vishal.glaitm@gmail.com, vinayk.deolia@gmail.com, tns11@yahoo.co.in
}

Received 3 January 2015; accepted 20 January 2015; published 27 January 2015

Copyright (C) 2015 by authors and Scientific Research Publishing Inc.

This work is licensed under the Creative Commons Attribution International License (CC BY). http://creativecommons.org/licenses/by/4.0/

(c) (i) Open Access

\section{Abstract}

This paper presents a robust sliding mode controller for a class of unknown nonlinear discretetime systems in the presence of fixed time delay. A neural-network approximation and the Lyapunov-Krasovskii functional theory into the sliding-mode technique is used and a neural-network based sliding mode control scheme is proposed. Because of the novality of Chebyshev Neural Networks (CNNs), that it requires much less computation time as compare to multi layer neural network (MLNN), is preferred to approximate the unknown system functions. By means of linear matrix inequalities, a sufficient condition is derived to ensure the asymptotic stability such that the sliding mode dynamics is restricted to the defined sliding surface. The proposed sliding mode control technique guarantees the system state trajectory to the designed sliding surface. Finally, simulation results illustrate the main characteristics and performance of the proposed approach.

\section{Keywords}

Discrete-Time Nonlinear Systems, Lyapunov-Krasovskii Functional, Linear Matrix Inequality (LMI), Sliding Mode Control (SMC), Chebyshev Neural Networks (CNNs)

\section{Introduction}

Time delay is undesirable parameter which is often encountered in various engineering systems, such as mechanical systems, chemical systems, and so on. The time delay degrades the system performance and leads to instability of the system. As a design tool for robust motion control system, SMC has been well designed for a wide range of nonlinear systems in both continuous time and discrete time. SMC is robust to parametric uncer- 
tainties and insensitive to unknown disturbance. SMC has been studied in seventies by the name of variable structure control (VSC) [1] [2]. VSC, is characterized by discontinuous feedback control law which switches the system in a predefined subspace [3]. Its implementation by a digital system requires sampling interval which leads to chattering. Over the past few decades, considerable attention has been reported to the stability analysis of continuous time delay systems by using different approaches [4]-[10]. In [4], robust controller has been designed for continuous time delay system using Ricacati equation approach. Considerable, attention has been given using LMI approach for stabilization of continuous time delay systems [5]-[8]. An adaptive control approach has been proposed for the control of time delay system [9] [10]. A discrete SMC (DSMC) is important when we implement robust control digitally with slow sampling rate. It is important to note that DSMC cannot be obtained from its continuous counterpart by simple conversion. Since modeling inaccuracy and external conditions lead to uncertainties, disturbances and nonlinearities in systems. Hence, the stability analysis of uncertain discrete-time delay systems have been studied over past few years with different control approaches have been well documented in [11] and reference therein. Moreover, in the above papers, the unknown nonlinearities have not been investigated. In [12], a robust control of uncertain nonlinear state delayed system, which gives a conservative condition of control, is presented. In recent years, many papers have reported the problem of SMC for state delay uncertain systems [13]-[17]. Most of these papers for uncertain time delay systems involve normbound nonlinearities which are treated as external disturbances. Adaptive multilayer neural control schemes for the control of complex nonlinear systems have shown great results over past few years. Now, it is an established fact that unknown nonlinear functions can be approximated from neural network. Neural network appears a powerful tool for nonlinear control problems [18]-[20]. In [21], the SMC have been used for control of uncertain state-delay system with unknown nonlinearity. In this work, Chebyshev Neural Network is used to estimate the unknown nonlinearity and linear matrix inequalities (LMI) conditions were derived to ensure the asymptotic stability on the defined sliding surface. Adaptive SMC for a class of discrete nonlinear systems was proposed. The proposed controller uses switching function with adaptive term to reduce the problem of chattering. Artificial neural network (ANN) was used for approximation of modeling errors. The nonlinearity is strictly positive and bounded away from zero [22]. In [23], a new SMC has been used to control the unknown nonlinear discrete-time systems. The chattering is reduced as compared to normal discrete-time sliding mode control using time varying gain.

This paper proposes a discrete-time sliding mode controller for a class of state delay nonlinear discrete systems. The unknown nonlinear functions in system dynamics is approximated using Chebyshev Neural Networks (CNNs). New weight update laws are derived to make this scheme adaptive. The stability of state delay system is taken care by carefully selecting Lyapunov-Krasovskii functional candidate. Thus conservative, sufficient conditions were derived which was represented by an appropriate set of LMIs.

The paper organization is as follows. Section 2 presents the CNN structure. Problem formulation and preliminaries are elaborated in Section 3. Section 4 presents controller design is stated in detail. The stability analysis is presented in Section 5. The effectiveness of proposed scheme is validates through simulation results in Section 6. The note ends with concluding remarks in Section 7.

Notations: $\|$.$\| denotes Euclidean norm, \|\|_{F}$ implies Frobenius norm. The $\operatorname{tr}($.$) stands for trace of matrix.$

\section{CNN Structure}

An ANN is a simple interconnected group of nonlinear elements, which has the capability to represents nonlinear functions. The representation accuracy depends on the ANN complexity, i.e., the number of elements and the way in which they are interconnected [24].

There is different ANN configuration available, like feed forward network such as multilayer perceptron (MLP), radial basis function (RBF) networks, Chebyshev neural network (CNN) etc. The MLP network has certain disadvantage that it requires a large amount of computation for learning. The RBF network can effectively learn from discontinuities and local variations. The problem with this network is choosing an appropriate set of RBF centers for effective learning. A single-layer functional link artificial neural network (FLANN) in which the need of hidden layer is eliminated by expanding the input pattern using Chebyshev polynomials. The main advantage of this network is that it requires much less computation as compared to a multilayer perceptron (MLP).

CNN is a functional link network (FLN) based on Chebyshev polynomials. CNN architecture has two main 
parts, namely, numerical transformation and learning [25]. In numerical transformation we use finite set of Chebyshev polynomials as a functional expansion (FE) of input pattern. The learning part is a functional-link neural network based on Chebyshev polynomials. The Chebyshev polynomials can be obtained by a recursive formula

$$
T_{i+1}(x)=2 x T_{i}(x)-T_{i-1}(x), \quad T_{0}(x)=1
$$

where, $T_{1}(x)$ are Chebyshev polynomials, $i$ is the order of polynomials chosen and here $x$ is a scalar quantity. The different choices of $T_{1}(x)$ are $x \& 2 x$.

The output of single layer neural network is given by

$$
\hat{g}(x)=\hat{w}^{\mathrm{T}} \phi
$$

where, $w$ are the weights and $\phi$ is the suitable basis function of neural network. Based on the approximation property of CNN [27]-[30], there exist ideal weights $w$, so that the function $g(x)$ to be approximated can be represented as

$$
g(x)=w^{\mathrm{T}} \phi+\varepsilon
$$

where, $\varepsilon$ is the CNN functional reconstruction error vector and $\|\varepsilon\| \leq \varepsilon_{N}$ is bounded.

\section{Problem Formulation}

Consider the following discrete-time state delay system as in [26] (Figure 1)

$$
x(k+1)=A x(k)+A_{d} x(k-h)+g(x(k)) u(k)
$$

where, $x(k) \in R^{n}$ and $u(k) \in R^{m}$ denote the state and input vectors respectively. $A$ and $A_{d}$ are real constant matrices with appropriate dimensions. $g(x(k))$ is a unknown nonlinear function of a given system in Equation (4), and $h$ is a positive number representing delay.

For the system given in (4) the sliding mode controller is obtained as

$$
u(k)=-\frac{1}{\hat{g}(x(k))}\left[A x(k)+A_{d} x(k-h)\right]
$$

where $\hat{g}(x(k))$ is the approximated value of the nonlinear function.

The objective of this work is to guarantee the stability of sliding mode controller in Equation (5) of the nonlinear system Equation (4), so that the system stays on the sliding surface.

\section{Controller Design}

The first step in the design of discrete-time SMC control algorithm would be the design of sliding surface. The linear sliding surface is defined as:

$$
s(k)=C x(k)
$$

where $C \in R^{m \times n}$ is a real matrix of appropriate dimensions.

For a system to be asymptotically stable, the sliding surface is defined as follows.

$$
s(k)=0
$$

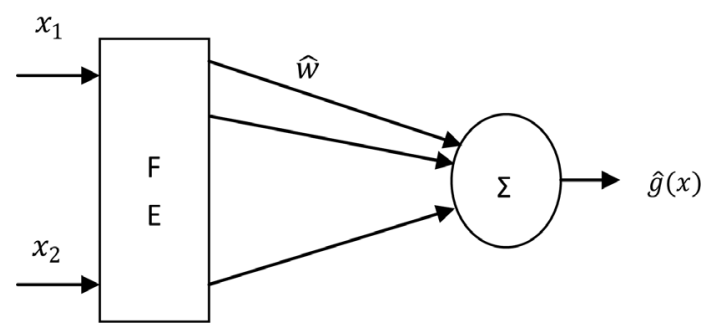

Figure 1. Chebyshev neural network. 
The second step is to design a control law which can guarantee the sliding mode reaching condition of the given linear sliding surface. The obtained control law is given in Equation (5) will force the trajectory of the system to move towards the sliding surface monotonically and causes zigzag motion around the sliding surface.

\section{Stability Analysis}

The following assumptions are needed for the stability analysis of the given unknown nonlinear system [26].

Assumption 1: The state delay $\mathrm{h}$ is a constant time delay that is basically induced by the network transmission. For constant time delay the lower and upper bounds are assumed to be identical.

Assumption 2: The nonlinear function $g(x(k))$ in the system is unknown and bounded.

Assumption 3: (Bounded Ideal NN Weights): The ideal NN weights $w$ are bounded so that $\|w\| \leq w_{M}$, with $w_{M}$ a known bound. The symbol \|\|$_{F}$ denotes the Frobenius norm, i.e. given a matrix $A$, the Frobenius norm is given by,

$$
\|A\|_{F}^{2}=\operatorname{tr}\left(A^{\mathrm{T}} A\right)
$$

Assumption 4: Let $\tilde{g}(x(k))=G \hat{g}(x(k))$, where $G=G^{\mathrm{T}}$ is a $n \times n$ symmetric matrix, and $\tilde{g}(x(k))$ and $\hat{g}_{x}(k)$ are the $n$-column vectors.

\section{Theorem 1:}

Given the system in Equation (4) and Assumptions 1 - 4, sliding mode control law Equation (5), the estimated NN weights are given by

$$
\hat{w}(k+1)=\left[(\hat{w}(k))+\left(x^{\mathrm{T}}(k)(Q-P-Z) x(k)\right)^{1 / 2}+\left(\hat{M}^{\mathrm{T}}(x(k)) Z \hat{M}(x(k))\right)^{1 / 2}\right]
$$

with the condition are

$$
\begin{gathered}
4\left((\hat{w}(k))\left(\hat{M}^{\mathrm{T}}(x(k)) Z \hat{M}(x(k))\right)^{1 / 2}\left(x^{\mathrm{T}}(k)(Q-P-Z) x(k)\right)^{1 / 2}\right)^{1 / 2}>0 \\
2\left(x^{\mathrm{T}}(k)(Q-P-Z) x(k)\right)^{1 / 2}\left(\hat{M}^{\mathrm{T}}(x(k)) Z \hat{M}(x(k))\right)^{1 / 2}>0
\end{gathered}
$$

Suppose there exist an $n \times n$ positive-definite matrix $P$, an $n \times n$ nonnegative-definite matrix $Q$, an $n \times n$ nonnegative-definite matrix $z$ and $n \times n$ symmetric matrix $G$ such that following LMI holds,

$$
H 1)=\left[\begin{array}{cc}
A^{\mathrm{T}} G^{\mathrm{T}} P G A-A^{\mathrm{T}} G^{\mathrm{T}} z G A-A^{\mathrm{T}} G^{\mathrm{T}} z-z G A & A^{\mathrm{T}} G^{\mathrm{T}} P G A_{d}-A^{\mathrm{T}} G^{\mathrm{T}} z G A_{d}-G z A_{d} \\
* & A_{d}^{\mathrm{T}} G^{\mathrm{T}} P G A_{d}-A_{d}^{\mathrm{T}} G^{\mathrm{T}} z G A_{d}-Q
\end{array}\right]<0
$$

Thus by properly selecting the control gain and the design parameters, the state trajectory is reaching on the designed sliding surface.

Proof: Choose Lyapunov-Krasovskii functional candidate,

$$
V(k)=V_{1}(k)+V_{2}(k)+V_{3}(k)+V_{4}(k)
$$

where

$$
\begin{aligned}
V_{1}(k) & =x^{\mathrm{T}}(k) P x(k) \\
V_{2}(k) & =\sum_{i=k-h}^{k-1} x^{\mathrm{T}}(i) Q x(i) \\
V_{3}(k) & =\operatorname{tr}\left(\tilde{w}^{\mathrm{T}}(k) \tilde{w}(k)\right) \\
V_{4}(k) & =\eta^{\mathrm{T}}(k) Z \eta(k)
\end{aligned}
$$

And 


$$
\eta(k)=x(k+1)-x(k)
$$

Substituting Equations (13)-(16) in Equation (12)

$$
V(k)=x^{\mathrm{T}}(k) P x(k)+\sum_{i=k-h}^{k-1} x^{\mathrm{T}}(i) Q x(i)+\operatorname{tr}\left(\tilde{w}^{\mathrm{T}}(k) \tilde{w}(k)\right)+\eta^{\mathrm{T}}(k) Z \eta(k)
$$

and

$$
V(k+1)=x^{\mathrm{T}}(k+1) P x(k+1)+\sum_{i=k-h+1}^{k} x^{\mathrm{T}}(i) Q x(i)+\operatorname{tr}\left(\tilde{w}^{\mathrm{T}}(k+1) \tilde{w}(k+1)\right)+\widehat{M}^{\mathrm{T}}(x(k)) Z \bar{M}(x(k))
$$

where

$$
\eta(k+1)=\hat{M}(x(k))
$$

Since $P, Z$ is a positive-definite and $Q$ is a nonnegative-definite, $V(k)$ is then positive-definite. Therefore,

$$
\Delta V(k)=V(k+1)-V(k)
$$

Substituting Equation (18) and (19) in Equation (21),

$$
\begin{aligned}
\Delta V(k)= & x^{\mathrm{T}}(k+1) P x(k+1)+\sum_{i=k-h+1}^{k} x^{\mathrm{T}}(i) Q x(i)+\operatorname{tr}\left(\tilde{w}^{\mathrm{T}}(k+1) \tilde{w}(k+1)\right)+\hat{M}^{\mathrm{T}}(x(k)) Z \hat{M}(x(k)) \\
& -x^{\mathrm{T}}(k) P x(k)-\sum_{i=k-h}^{k-1} x^{\mathrm{T}}(i) Q x(i)-\operatorname{tr}\left(\tilde{w}^{\mathrm{T}}(k) \tilde{w}(k)\right)-\eta^{\mathrm{T}}(k) Z \eta(k) .
\end{aligned}
$$

Substituting Equation (5) in Equation (21) and using Assumption 3 in Equation (22)

$$
\begin{aligned}
\Delta V(k)= & {\left[A x(k)+A_{d} x(k-h)+\tilde{g}(x(k)) u(k)+\hat{g}(x(k)) u(k)\right] } \\
& \times P\left[A x(k)+A_{d} x(k-h)+\tilde{g}(x(k)) u(k)+\hat{g}(x(k)) u(k)\right] \\
& +x^{\mathrm{T}}(k) Q x(k)+\hat{M}^{\mathrm{T}}(x(k)) Z \hat{M}(x(k))-x^{\mathrm{T}}(k) P x(k)-x^{\mathrm{T}}(k-h) Q x(k-h) \\
& -[x(k+1)-x(k)]^{\mathrm{T}} Z[x(k+1)-x(k)]+\left\|\tilde{w}^{\mathrm{T}}(k+1)\right\|^{2}-\left\|\tilde{w}^{\mathrm{T}}(k)\right\|^{2} .
\end{aligned}
$$

After some mathematical manipulations in Equation (23),

$$
\begin{aligned}
\Delta V(k)= & x^{\mathrm{T}}(k) Q x(k)+\hat{M}^{\mathrm{T}}(x(k)) Z \hat{M}(x(k))-x^{\mathrm{T}}(k) P x(k)-x^{\mathrm{T}}(k-h) Q x(k-h)-x^{\mathrm{T}}(k) Z x(k) \\
& +\left\|\tilde{w}^{\mathrm{T}}(k+1)\right\|^{2}-\left\|\tilde{w}^{\mathrm{T}}(k)\right\|^{2}+Z(k) .
\end{aligned}
$$

where

$$
\begin{aligned}
Z(k)= & x^{\mathrm{T}}(k) A^{\mathrm{T}} P \tilde{g}(x(k)) u(k)+x^{\mathrm{T}}(k-h) A_{d}^{\mathrm{T}} P \tilde{g}(x(k)) u(k)+u^{\mathrm{T}}(k) \hat{g}^{\mathrm{T}}(x(k)) P \tilde{g}(x(k)) \\
& +u^{\mathrm{T}}(k) \tilde{g}^{\mathrm{T}}(x(k)) P A x(k)+u^{\mathrm{T}}(k) \tilde{g}^{\mathrm{T}}(x(k)) P A_{d}^{\mathrm{T}} x(k-h)+u^{\mathrm{T}}(k) \tilde{g}^{\mathrm{T}}(x(k)) P \hat{g}(x(k)) u(k) \\
& +u^{\mathrm{T}}(k) \tilde{g}^{\mathrm{T}}(x(k)) P \tilde{g}(x(k)) u(k)-x^{\mathrm{T}}(k) A^{\mathrm{T}} Z \tilde{g}(x(k)) u(k)-x^{\mathrm{T}}(k-h) A_{d}^{\mathrm{T}} Z \tilde{g}(x(k)) u(k) \\
& -u^{\mathrm{T}}(k) \hat{g}^{\mathrm{T}}(x(k)) Z \tilde{g}(x(k))-u^{\mathrm{T}}(k) \tilde{g}^{\mathrm{T}}(x(k)) Z A x(k)-u^{\mathrm{T}}(k) \tilde{g}^{\mathrm{T}}(x(k)) Z A_{d}^{\mathrm{T}} x(k-h) \\
& -u^{\mathrm{T}}(k) \tilde{g}^{\mathrm{T}}(x(k)) Z \hat{g}(x(k)) u(k)-u^{\mathrm{T}}(k) \tilde{g}^{\mathrm{T}}(x(k)) Z \tilde{g}(x(k)) u(k)+u^{\mathrm{T}}(k) \tilde{g}^{\mathrm{T}}(x(k)) Z x(k) \\
& +x^{\mathrm{T}}(k) Z \tilde{g}(x(k)) u(k) .
\end{aligned}
$$

Collecting the terms together and substitute control law Equation (5) in Equation (24) yields

$$
\begin{aligned}
\Delta V(k)= & -\|\hat{w}(k+1)\|^{2}+\left[(\hat{w}(k))+\left(x^{\mathrm{T}}(k)(Q-P-Z) x(k)\right)^{1 / 2}+\left(\hat{M}^{\mathrm{T}}(x(k)) Z \hat{M}(x(k))\right)^{1 / 2}\right]^{2} \\
& +S(k)+Z(k)
\end{aligned}
$$

where 


$$
\begin{aligned}
S(k)= & -2(\hat{w}(k))\left(\hat{M}^{\mathrm{T}}(x(k)) Z \hat{M}(x(k))\right)^{1 / 2}-2(\hat{w}(k))\left(x^{\mathrm{T}}(k)(Q-P-Z) x(k)\right)^{1 / 2} \\
& -2\left(x^{\mathrm{T}}(k)(Q-P-Z) x(k)\right)^{1 / 2}\left(\hat{M}^{\mathrm{T}}(x(k)) Z \hat{M}(x(k))\right)^{1 / 2}-x^{\mathrm{T}}(k-h) Q x(k-h) .
\end{aligned}
$$

with tuning law in Equation (8), Equation (26) will be,

$$
\Delta V(k)=S(k)+Z(k)
$$

substituting Equations (25), (27) in Equation (28)

$$
\begin{aligned}
\Delta V(k)= & -2(\hat{w}(k))\left(\hat{M}^{\mathrm{T}}(x(k)) Z \hat{M}(x(k))\right)^{1 / 2}-2(\hat{w}(k))\left(x^{\mathrm{T}}(k)(Q-P-Z) x(k)\right)^{1 / 2} \\
& -2\left(x^{\mathrm{T}}(k)(Q-P-Z) x(k)\right)^{1 / 2}\left(\hat{M}^{\mathrm{T}}(x(k)) Z \hat{M}(x(k))\right)^{1 / 2} \\
& +x^{\mathrm{T}}(k)\left[A^{\mathrm{T}} G^{\mathrm{T}} P G A-A^{\mathrm{T}} G^{\mathrm{T}} Z G A-A^{\mathrm{T}} G^{\mathrm{T}} Z-Z G A\right] x(k) \\
& +x^{\mathrm{T}}(k)\left[A^{\mathrm{T}} G^{\mathrm{T}} P G A_{d}-A^{\mathrm{T}} G^{\mathrm{T}} Z G A_{d}-Z G A_{d}\right] x(k-h) \\
& +x^{\mathrm{T}}(k-h)\left[A_{d}^{\mathrm{T}} G^{\mathrm{T}} P G A-A_{d}^{\mathrm{T}} G^{\mathrm{T}} Z G A-A_{d}^{\mathrm{T}} Z G^{\mathrm{T}}\right] x(k) \\
& +x^{\mathrm{T}}(k-h)\left[A_{d}^{\mathrm{T}} G^{\mathrm{T}} P G A_{d}-A_{d}^{\mathrm{T}} G^{\mathrm{T}} Z G A_{d}-Q\right] x(k-h) .
\end{aligned}
$$

Manipulating the nonquadratic terms using the following inequality $\sqrt{a b} \leq \frac{a+b}{2}$ (which turns into equality if and only if $a=b$ we get,

$$
\begin{aligned}
\Delta V(k) \leq & -4\left((\hat{w}(k))\left(\hat{M}^{\mathrm{T}}(x(k)) Z \hat{M}(x(k))\right)^{1 / 2}\left(x^{\mathrm{T}}(k)(Q-P-Z) x(k)\right)^{1 / 2}\right)^{1 / 2} \\
& -2\left(x^{\mathrm{T}}(k)(Q-P-Z) x(k)\right)^{1 / 2}\left(\hat{M}^{\mathrm{T}}(x(k)) Z \hat{M}(x(k))\right)^{1 / 2} \\
& +x^{\mathrm{T}}(k)\left[A^{\mathrm{T}} G^{\mathrm{T}} P G A-A^{\mathrm{T}} G^{\mathrm{T}} Z G A-A^{\mathrm{T}} G^{\mathrm{T}} Z-Z G A\right] x(k) \\
& +x^{\mathrm{T}}(k)\left[A^{\mathrm{T}} G^{\mathrm{T}} P G A_{d}-A^{\mathrm{T}} G^{\mathrm{T}} Z G A_{d}-Z G A_{d}\right] x(k-h) \\
& +x^{\mathrm{T}}(k-h)\left[A_{d}^{\mathrm{T}} G^{\mathrm{T}} P G A-A_{d}^{\mathrm{T}} G^{\mathrm{T}} Z G A-A_{d}^{\mathrm{T}} Z G^{\mathrm{T}}\right] x(k) \\
& +x^{\mathrm{T}}(k-h)\left[A_{d}^{\mathrm{T}} G^{\mathrm{T}} P G A_{d}-A_{d}^{\mathrm{T}} G^{\mathrm{T}} Z G A_{d}-Q\right] x(k-h) .
\end{aligned}
$$

where

$$
\begin{gathered}
a=-2(\hat{w}(k))\left(\hat{M}^{\mathrm{T}}(x(k)) Z \hat{M}(x(k))\right)^{1 / 2} \\
b=-2(\hat{w}(k))\left(x^{\mathrm{T}}(k)(Q-P-Z) x(k)\right)^{1 / 2}
\end{gathered}
$$

In Equation (30), $\Delta V(k)$ is guaranteed to remain negative as long as

$$
\left[\begin{array}{cc}
A^{\mathrm{T}} G^{\mathrm{T}} P G A-A^{\mathrm{T}} G^{\mathrm{T}} z G A-A^{\mathrm{T}} G^{\mathrm{T}} z-z G A & A^{\mathrm{T}} G^{\mathrm{T}} P G A_{d}-A^{\mathrm{T}} G^{\mathrm{T}} z G A_{d}-G z A_{d} \\
* & A_{d}^{\mathrm{T}} G^{\mathrm{T}} P G A_{d}-A_{d}^{\mathrm{T}} G^{\mathrm{T}} z G A_{d}-Q
\end{array}\right]<0
$$

Since the first two terms in Equation (30) are satisfying the condition in Equation (9), (10), next four terms are satisfying the LMI in Equation (11). Therefore, we conclude that the system in Equation (4) is stable with control law Equation (5) and LMI in Equation (11).

\section{Simulation Results}

In this section, a numerical example is presented to validate the performance and effectiveness of the nonlinear discrete-time system proposed in Equation (4). Consider the set of parameters for the given system

$$
A=\left[\begin{array}{cc}
0.8 & 0.4 \\
-0.14 & 0.1
\end{array}\right], \quad A_{d}=\left[\begin{array}{cc}
0.2 & 0 \\
0 & 0.1
\end{array}\right]
$$


and

$$
g(x(k))=\left[\begin{array}{c}
\frac{1.4 x_{1}^{2}(k)}{1+x_{1}^{2}(k)} \\
\frac{x_{1}(k)}{1+x_{1}^{2}(k)+x_{2}^{2}(k)}
\end{array}\right]
$$

The fixed time delay is assumed to be $h=2$. The proposed system has initial condition of states $x_{1}$ and $x_{2}$ are chosen as $[0.01 \quad 0,02]^{\mathrm{T}}$. The LMI in H1) are solved by using Matlab LMI Toolbox and the values of $P$, $Q, Z$ and $G$ are obtained as

$$
\begin{array}{cc}
P=\left[\begin{array}{cc}
108.8411 & 0 \\
0 & 108.8411
\end{array}\right], \quad Q=10^{-11}\left[\begin{array}{cc}
0.1040 & 0.0338 \\
0.0338 & -0.0186
\end{array}\right] \\
G=10^{-10}\left[\begin{array}{cc}
-0.1198 & 0.0001 \\
0.0001 & 0.0247
\end{array}\right], \quad z=10^{-11}\left[\begin{array}{cc}
0.3485 & -0.0103 \\
-0.0103 & 0.4639
\end{array}\right]
\end{array}
$$

The trajectories of the system states $x_{1}$ and $x_{2}$ are shown in Figure 2 and Figure 3. It is observed in Figure 2 and Figure 3 that the states of the sliding motion approach to zero quickly. Figure 4 demonstrates that the controller robustly stabilizes the system by sliding mode technique with fixed time delay. The simulation results confirm the stability of the system and show the effectiveness of proposed scheme.

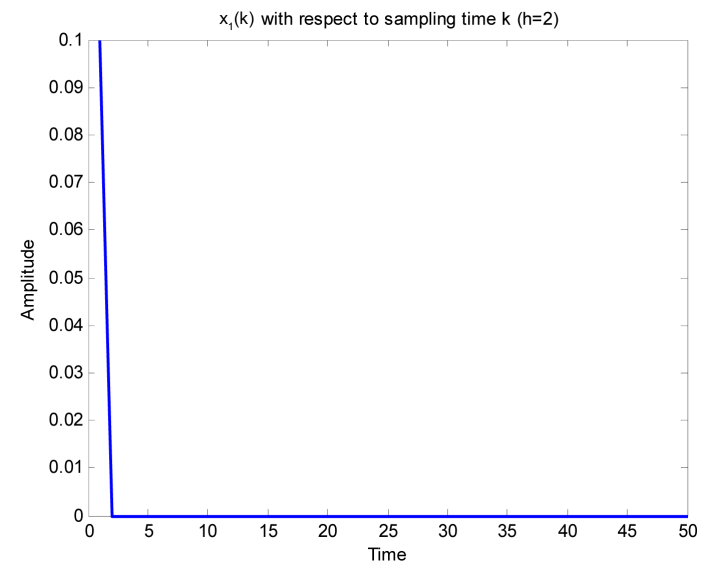

Figure 2. $x_{1}(k)$ with respect to sample time $k \quad(h=2)$.

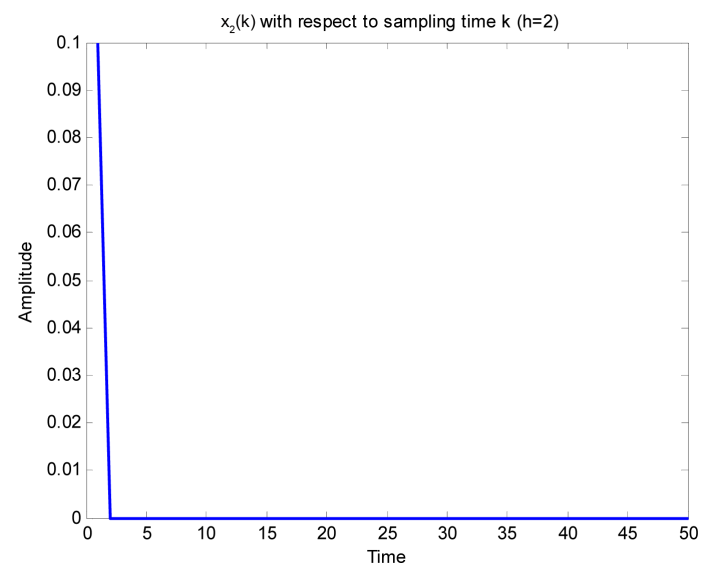

Figure 3. $x_{2}(k)$ with respect to sample time $k \quad(h=2)$. 


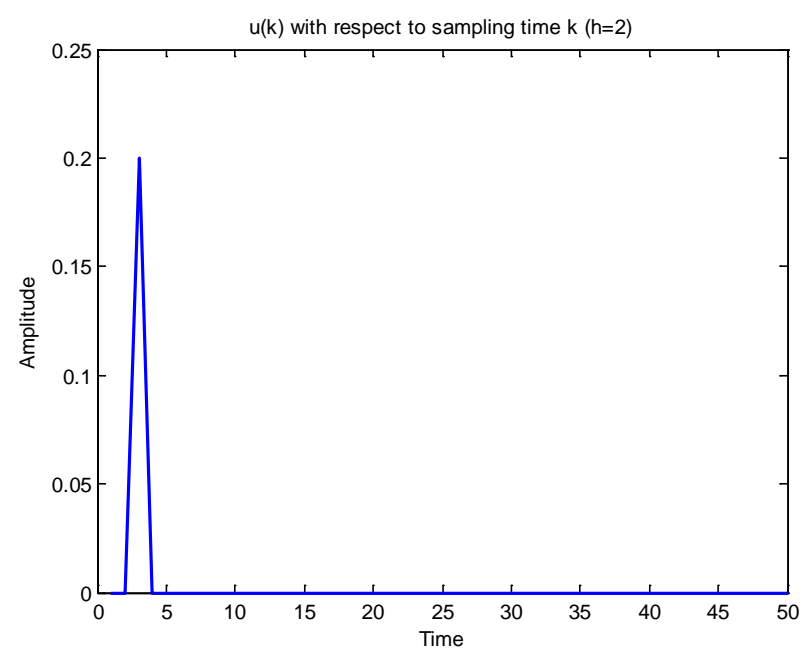

Figure 4. $u(k)$ with respect to sample time $k \quad(h=2)$.

\section{Conclusion}

In this paper, a sliding mode control for a class of unknown nonlinear discrete-time system is proposed ,which results in small chattering motion in both control signal and system output. A Chebyshev Neural Network is used to approximate the unknown system dynamics. A new learning algorithm for neural network approximation is proposed. This neural network based sliding mode control approach guarantees the system state trajectory to the defined sliding surface. An LMI based sufficient condition for the asymptotic stability of the sliding mode dynamics is derived by means of a Lyapunov-Krasovskii approach. Simulation results are validating the effectiveness of proposed scheme.

\section{References}

[1] Utkin, V.I. (1977) Variable Structure Systems with Sliding Modes. IEEE Transactions on Automatic Control, AC-22, 212-222. http://dx.doi.org/10.1109/TAC.1977.1101446

[2] Utkin, V.I. (1978) Sliding Modes and Their Applications in Variable Structure Systems. Nauka, Moscow.

[3] Sarpturk, S.Z., Istefanopolos, Y. and Kaynak, O. (1987) On the Stability of Discrete-Time Sliding Mode Control Systems. IEEE Transactions on Automatic Control, 32, 930-932. http://dx.doi.org/10.1109/TAC.1987.1104468

[4] Jeung, E.T., Oh, D.C., Kim, J.H. and Park, H.B. (1996) Robust Controller Design for Uncertain Systems with Time Delays, LMI Approach. Automatica, 32, 1229-1231. http://dx.doi.org/10.1016/0005-1098(96)00055-6

[5] Yue, D. (2004) Robust Stabilization of Uncertain Systems with Unknown Input Delay. Automatica, 40, 331-336. http://dx.doi.org/10.1016/j.automatica.2003.10.005

[6] Basin, M.V., Perez, J., Acosta, P. and Fridman, L. (2006) Optimal Filtering for Nonlinear Polynomial Systems over Linear Observations with Delay. International Journal of Innovative Computing Information and Control, 2, 863-874.

[7] Boukas, E.K. and Al-Muthairi, N.F. (2006) Delay-Dependent Stabilization of Singular Linear Systems with Delays. International Journal of Innovative Computing Information and Control, 2, 283-291.

[8] Chen, M., Lam, J. and Xu, S. (2006) Memory State Feedback Guaranteed Cost Control for Neutral Delay Systems. International Journal of Innovative Computing Information and Control, 2, 293-303

[9] Ge, S.S., Hong, F. and Lee, T.H. (2004) Adaptive Neural Control of Nonlinear Time-Delay Systems with Unknown Virtual Control Coefficients. IEEE Transactions on Systems, Man, and Cybernetics, Part B: Cybernetics, 34, 449-516. http://dx.doi.org/10.1109/TSMCB.2003.817055

[10] Ge, S.S., Hong, F. and Lee, T.H. (2003) Adaptive Neural Network Control of Nonlinear Systems with Unknown Time Delays. IEEE Transactions on Automatic Control, 48, 2004-2010. http://dx.doi.org/10.1109/TAC.2003.819287

[11] Xia, Y. and Jia, Y. (2003) Robust Sliding-Mode Control for Uncertain Time-Delay Systems: An LMI Approach. IEEE Transactions on Automatic Control, 48, 1086-1092. http://dx.doi.org/10.1109/TAC.2003.812815

[12] Wang, Z., Huang, B. and Unbehauen, H. (1999) Robust Reliable Control for a Class of Uncertain Nonlinear State-Delayed System. Automatica, 35, 955-963. http://dx.doi.org/10.1016/S0005-1098(98)00233-7 
[13] Shyu, K. and Yan, J. (1993) Robust Stability of Uncertain Time-Delay Systems and Its Stabilization by Variable Structure Control. International Journal of Control, 57, 237-246. http://dx.doi.org/10.1080/00207179308934385

[14] Roh, Y.H. and Oh, J.H. (1999) Robust Stabilization of Uncertain Input-Delay Systems by Sliding Mode Control with Delay Compensation. Automatica, 35, 1861-1865. http://dx.doi.org/10.1016/S0005-1098(99)00106-5

[15] Basin, M.V., Gonzalez, J.R. and Fridman, L. (2003) Optimal and Robust Sliding Mode Control for Linear Systems with Time Delays in Control Input. International Journal of Pure and Applied Mathematics, 5, 395-420.

[16] Xia, Y., Liu, G.P., Shi, P., Chen, J. and Rees, D. (2008) Robust Delay-Dependent Sliding Mode Control for Uncertain Time-Delay Systems. International Journal of Robust and Nonlinear Control, 18, 1142-1161. http://dx.doi.org/10.1002/rnc.1272

[17] Yan, M. and Shi, Y. (2008) Robust Discrete-Time Sliding Mode Control for Uncertain Systems with Time-Varying State Delay. IET Control Theory \& Applications, 2, 662-674. http://dx.doi.org/10.1049/iet-cta:20070460

[18] Zhang, T., Ge, S.S. and Hang, C.C. (1999) Design and Performance Analysis of a Direct Adaptive Controller for Nonlinear Systems. Automatica, 35, 1809-1817. http://dx.doi.org/10.1016/S0005-1098(99)00098-9

[19] Chen, S. and Billings, S.A. (1992) Neural Networks for Nonlinear Dynamic System Modelling and Identification. International Journal of Control, 56, 319-346. http://dx.doi.org/10.1080/00207179208934317

[20] Jagannathan, S. and Lewis, F.L. (1996) Identification of Nonlinear Dynamical Systems Using Multilayered Neural Networks. Automatica, 36, 1707-1712. http://dx.doi.org/10.1016/S0005-1098(96)80007-0

[21] Niu, Y., Lam, J., Wang, X. and Ho, D.W.C. (2003) Sliding-Mode Control for Nonlinear State-Delayed Systems Using Neural-Network Approximation. IEEE Proceedings_Control Theory and Applications, 150, 233-239.

[22] Munoz, D. and Sbarbaro, D. (2000) An Adaptive Sliding-Mode Controller for Discrete Nonlinear Systems. IEEE Transaction on Industrial Electronics, 47, 574-581. http://dx.doi.org/10.1109/41.847898

[23] de Jesús Rubio, J. and Yu, W. (2006) Discrete-Time Sliding-Mode Control Based on Neural-Networks. Springer-Verlag, Berlin, 956-961.

[24] Patra, J.C. and Kot, A.C. (2002) Nonlinear Dynamic System Identification Using Chebyshev Functional Link Artificial Neural Networks. IEEE Transaction on Systems, Man and Cyberntics, 32, 505-511.

[25] Purwar, S., Kar, I.N. and Jha, A.N. (2007) Nonlinear System Identification Using Neural Networks. IETE Journal of Research, 53, 35-42. http://dx.doi.org/10.1080/03772063.2007.10876119

[26] Deolia, V.K., Purwar, S. and Sharma, T.N. (2012) Stabilization of Unknown Nonlinear Discrete-Time Delay Systems Based on Neural Network. Intelligent Control and Automation, 3, 337-345.

[27] Namatame, A. and Ueda, N. (1992) Pattern Classification with Chebyshev Neural Network. International Journal Neural Network, 3, 23-31.

[28] Lee, T.T. and Jeng, J.T. (1998) The Chebyshev Polynomial Based Unified Model Neural Networks for Functions Approximations. IEEE Transactions on Systems, Man \& Cybernetics, Part B, 28, 925-935. http://dx.doi.org/10.1109/3477.735405

[29] Purwar, S., Kar, I.N. and Jha, A.N. (2008) Adaptive Output Feedback Tracking Control of Robot Manipulators Using Position Measurements Only. Expert Systems with Applications, 34, 2789-2798. http://dx.doi.org/10.1016/j.eswa.2007.05.030

[30] Purwar, S., Kar, I.N. and Jha, A.N. (2005) On-Line System Identification of Complex Systems Using Chebyshev Neural Networks. Applied Soft Computing, 7, 364-372. http://dx.doi.org/10.1016/j.asoc.2005.08.001 
Scientific Research Publishing (SCIRP) is one of the largest Open Access journal publishers. It is currently publishing more than 200 open access, online, peer-reviewed journals covering a wide range of academic disciplines. SCIRP serves the worldwide academic communities and contributes to the progress and application of science with its publication.

Other selected journals from SCIRP are listed as below. Submit your manuscript to us via either submit@scirp.org or Online Submission Portal.
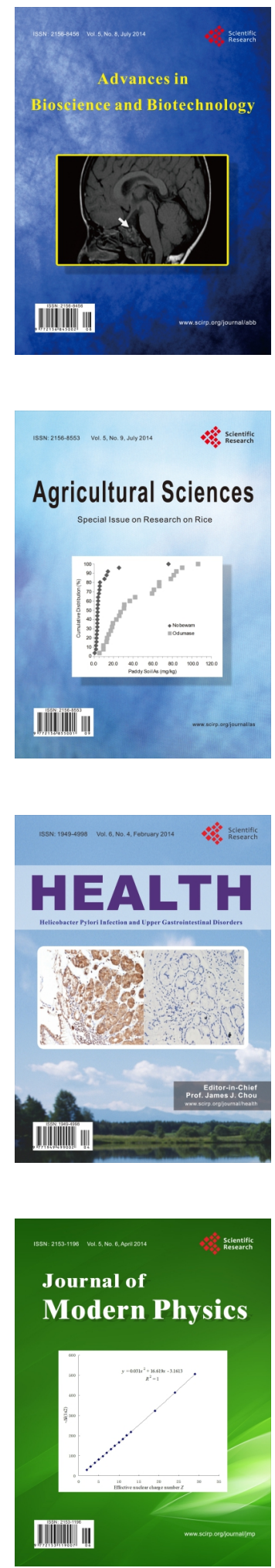
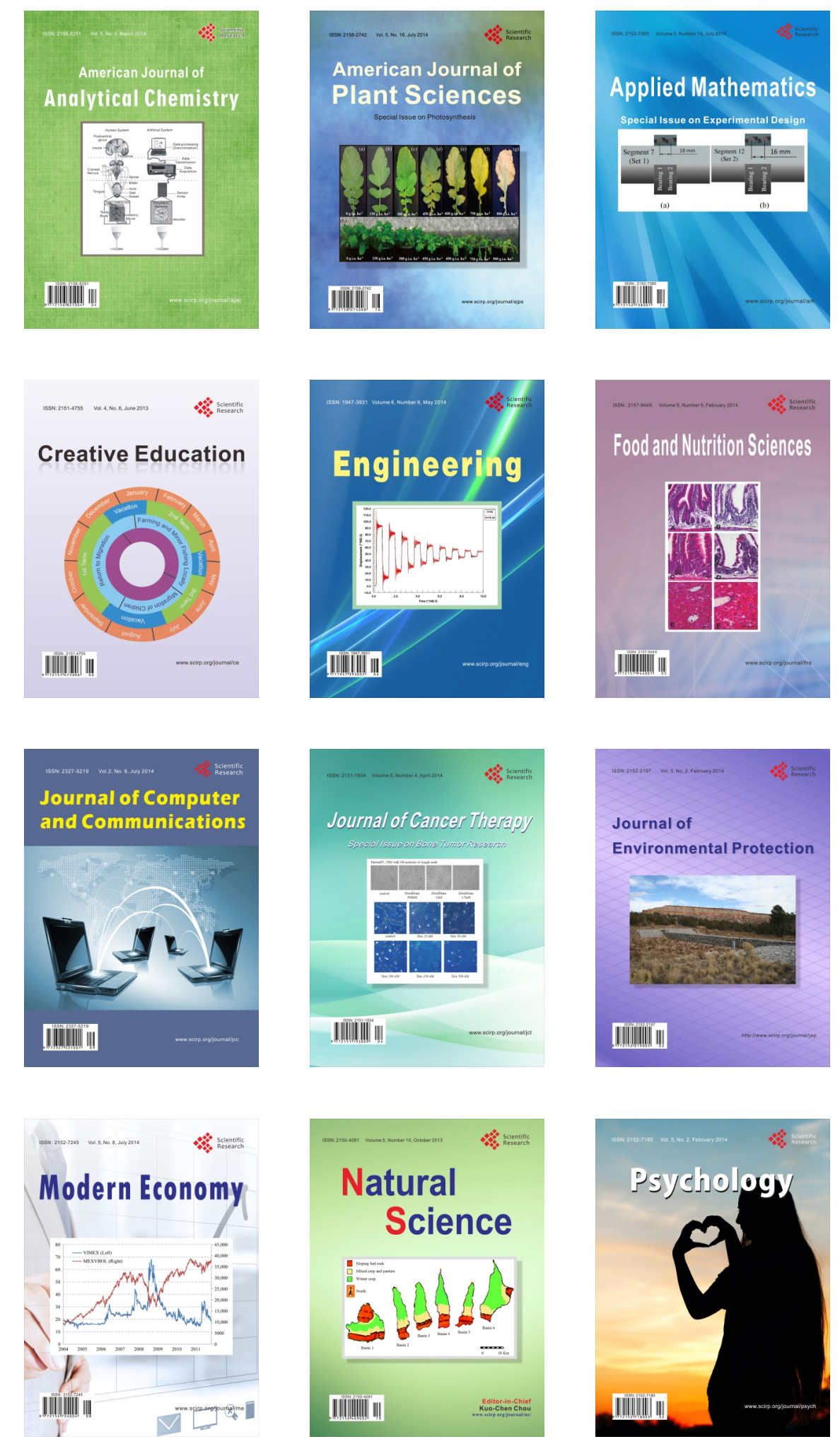